INSTITUTE OF FORESTRY • BELGRADE

INSTITUT ZA ŠUMARSTVO • BEOGRAD

SUSTAINABLE FORESTRY

COLLECTION 69-70, 2014
ODRŽIVO ŠUMARSTVO

ZBORNIK RADOVA 69-70, 2014

UDK $630 * 181.45+630 * 425.1(497.11) " 2014 "=111$

Original scientific paper

\title{
INTENSIVE MONITORING ON THE LEVEL II SAMPLE PLOTS KOPAONIK, CRN VRH I MOKRA GORA IN 2014
}

\author{
Svetlana BILIBAJKIĆ, Radovan NEVENIĆ Miroslava MARKOVIĆ, \\ Goran $\breve{C} E \check{S} L J A R$, Tomislav STEFANOVIĆ, Zoran PODUŠKA, Ilija ĐORĐEVIĆ, \\ Renata GAGIĆ SERDAR ${ }^{1}$
}

\begin{abstract}
Monitoring and assessment of the impact of air pollution and its effects on forest ecosystems on the territory of the Republic of Serbia, Level II, began with the establishment of Level II sample plots on Fruska Gora in 2009, on Kopaonik in 2010, in Odzaci in 2011 and on Crni Vrh and Mokra Gora in 2013. Level II Monitoring Programme includes ten working panels from ten different professional fields of forestry grouped according to the research area. All Level II sample plot activities are carried out in accordance with the ICP Forests Manual on methods and criteria for harmonized sampling, assessment, monitoring and analysis of the effects of air pollution on forests. The paper presents the results of monitoring crown condition on Level II sample plots on Kopaonik, Crni Vrh and Mokra Gora in 2014.
\end{abstract}

Key words: Level II sample plot, crown condition, defoliation, damage, GIS.

\section{INTENZIVNI MONITORING NA OGLEDNIM POLJIMA BIT NIVO-a II KOPAONIK, CRNI VRH I MOKRA GORA U 2014.GODINI}

\begin{abstract}
Praćenje i procenu uticaja vazdušnih zagađenja i njihovih efekata u šumskim ekosistemima на mepumopuju Republike Srbije, Nivo II, otpočelo je osnivanjem bioindikacijskih tačka Nivo-a II i to 2009. godine na Fruškoj gori, 2010. godine na Kopaoniku, 2011. godine u Odžacima i 2013. godine na Crnom vrhu i Mokroj Gori. Program monitoringa Nivo-a II obuhvata deset radnih panela iz deset zasebnih stručnih oblasti šumarstva grupisanih prema predmetu istraživanja. Sve aktivnosti na BIT Nivo II sprovode se u skladu sa uputstvima o metodama $i$ kriterijumima za usaglašeno
\end{abstract}

\footnotetext{
${ }^{1}$ Institute of Forestry, Belgrade, Serbia

Translation: Dragana Ilić
} 
uzorkovanje, ocenu, monotoring $i$ analizu uticaja zagađenja vazduha na šume prema ICP Forests Manual-u. U radu su dati rezultati praćenja stanja kruna stabala na oglednim parcelama BIT Nivo-a II na Kopaoniku, Crnom vrhu i Mokroj Gori u 2014. godini.

Ključne reči: BIT Nivo II, stanje kruna, defolijacija, oštećenja, GIS.

\section{INTRODUCTION}

Level II monitoring of forest vitality is a system of applied comparative research studies that belong to different scientific fields of forestry. It is characterized by a more elaborate multidisciplinary approach. Level II measurements include an extremely greater number of parameters than the Level I measurements. Level II sample plots have been established throughout Europe according to the harmonized methodology of the ICP Forests programme. The primary aim of the programme is to make continuous measurements and to collect data on the state of forests that grow in different environmental conditions.

Serbia joined the European network of Level II sample plots when we established the sample plots in the NP Fruska Gora, NP Kopaonik, Odzaci, Crni Vrh and Mokra Gora.

\section{MATERIAL AND METHOD}

The intensive monitoring plot - Level II sample plot Kopaonik was established in 2010 in the National Park Kopaonik in a pure spruce stand, Picea abies (L.) H.Karst.
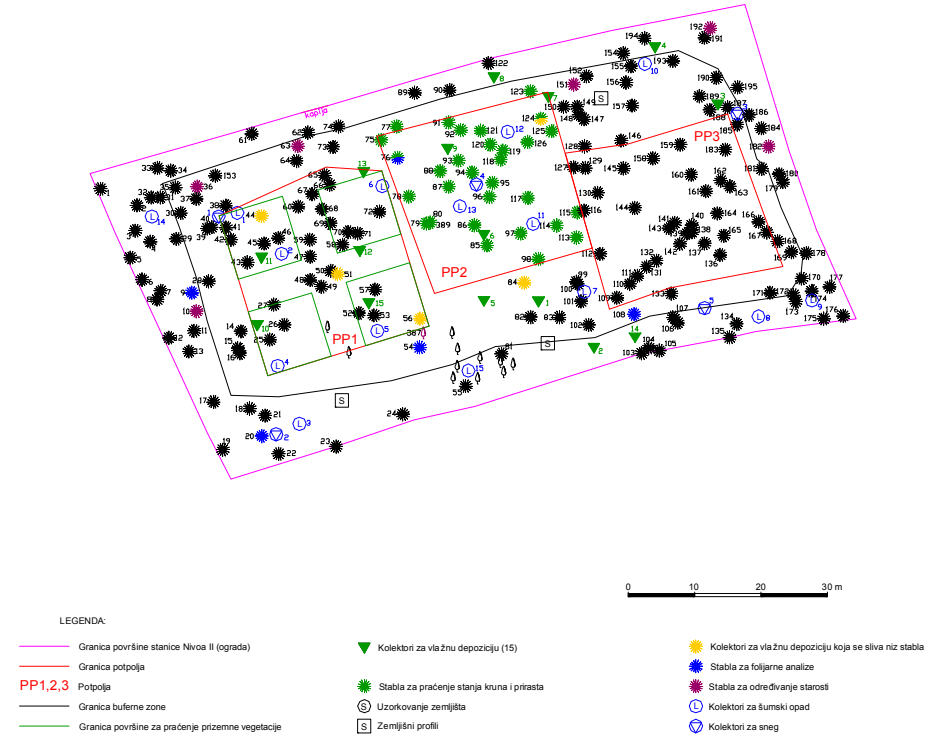

Figure 1. Field plan ${ }^{1}$ of the sample plot Kopaonik

\footnotetext{
${ }^{1}$ A digital field map was created at the Institute, according to the situation in the field and the initial draft of the sample subplots, created by a Faculty of Forestry team, in 2010.
} 
Another two Level II sample plots were established in 2013 one on Crni Vrh and the other in Mokra Gora.

The Level II sample plot on Crni Vrh was established in a pure stand of beech (Fagus moesiaca), while the Level II sample plot in Mokra Gora lies in an artificially- established stand of Scots pine (Pinus silvestris).

Each of the three Level II sample plots covers an area of 0.5 ha $(100 \times 50 \mathrm{~m})$, with three subplots, $25 \times 25 \mathrm{~m}$ in size, established in the area for the purpose of monitoring (a subplot intended for the crown condition assessment, phenology and and growth, a subplot for soil surveys and a subplot for ground vegetation assessment) and a buffer zone.

The plots are fenced with galvanized wire rope, two meters in height. The fence has two gates, one for vehicles $(3.0 \mathrm{~m}$ wide) and one for people $(1.5 \mathrm{~m}$ wide) .

The trees within the sample plots are permanently marked with numbers on the bark, and their position is located in the network.

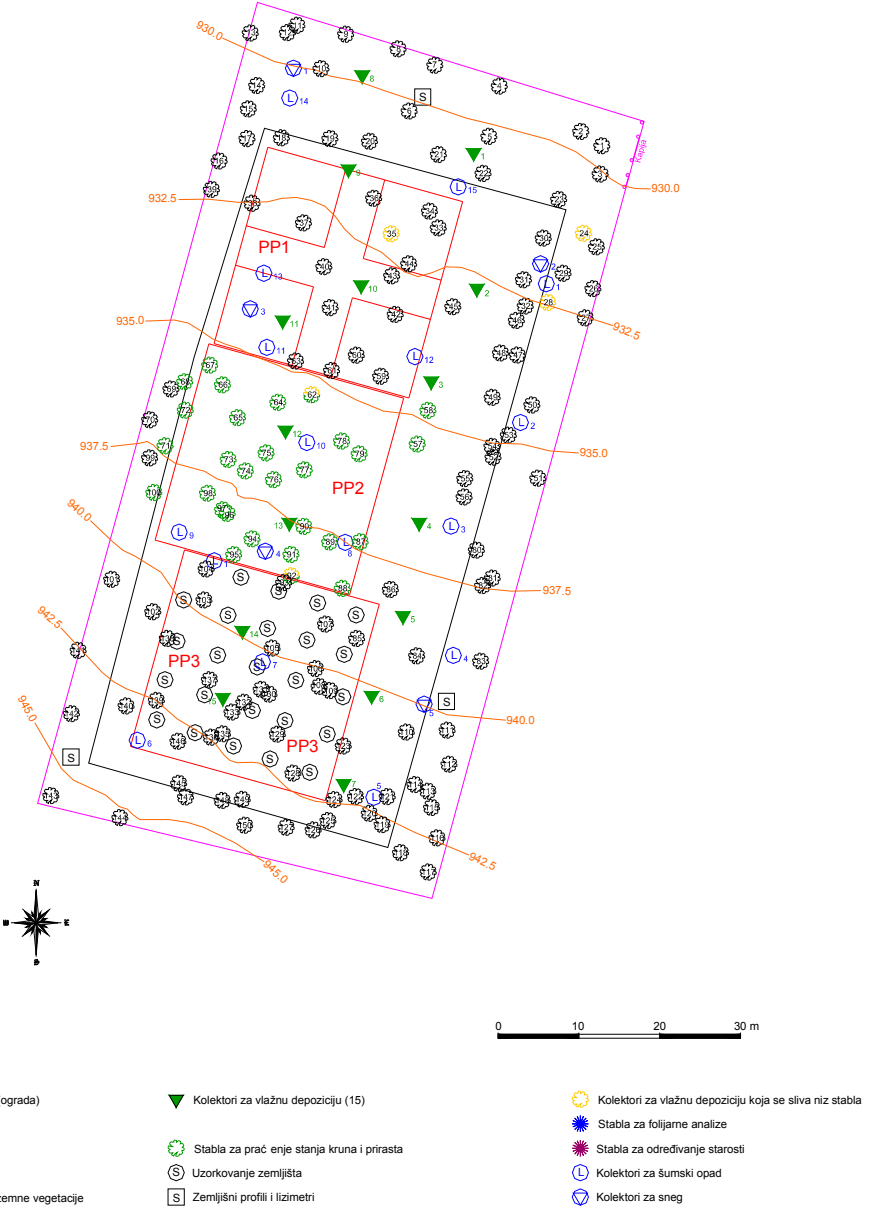

Figure 2. Digital field map ${ }^{l}$ of the sample plot Crni Vrh

\footnotetext{
${ }^{1}$ A digital field map was created at the Institute in accordance with the situation in the field
} 
The total number of trees within the Level II sample plot on Kopaonik is 195, while it amounts to 150 on Crni Vrh and 450 in Mokra Gora.

Digital field maps, which have been created for each sample plot, show the position of all trees and all measuring instruments as well as the altitude of the presented terrain (Figure 1,2 and 3).

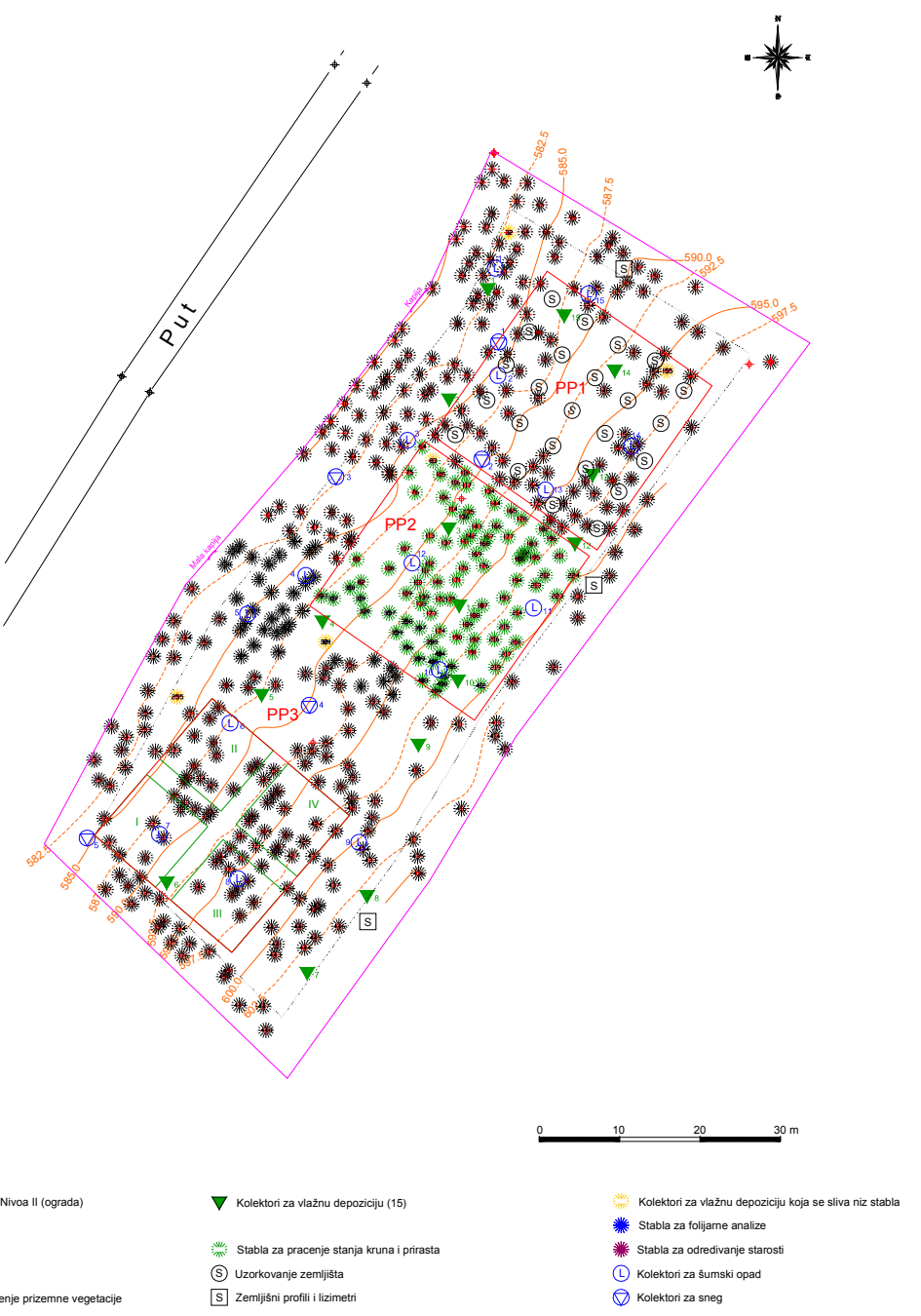

Figure 3. Digital field map ${ }^{1}$ of the sample plot Mokra Gora

The Level II monitoring programme includes the following groups of parameters: crown condition, foliar analyses, soil chemistry, soil solution chemistry, tree growth, ground vegetation, atmospheric depositions, ozone injuries,

\footnotetext{
${ }^{1}$ A digital field map was created at the Institute in accordance with the situation in the field
} 
meteorology, phenology, and litterfall. The frequency of parameter monitoring is shown in Table 1.

Table 1. Parameters, frequency and intensity of monitoring for Level II

\begin{tabular}{|l|l|}
\hline \multicolumn{1}{|c|}{ Parameter } & \multicolumn{1}{c|}{ Monitoring frequency } \\
\hline Crown condition & At least annually \\
\hline Foliar analyses & Every two year \\
\hline Soil chemistry & Every ten years \\
\hline Soil solution chemistry & Continuously \\
\hline Tree growth & Every five years \\
\hline Ground vegetation & Every five years \\
\hline Atmospheric deposition & Continuously \\
\hline Air quality & Continuously \\
\hline Ozone injury & Annually \\
\hline Meteorology & Continuously \\
\hline Phenology & Several times a year \\
\hline Forest litter & Continuously \\
\hline
\end{tabular}

\section{RESULTS AND DISCUSSION}

The improved methodological approach to the Level II crown condition assessment can be described as a set of characteristics of dominant tree crowns that are monitored in a similar way. The intensive monitoring scores (Nevenic et al., 2011), obtained for each individual tree whose crown is monitored every year, will after a certain number of replications give answers to different hypothetical assumptions, such as the causes of serious deterioration of forest vitality by identifying the agents and defining their harmfulness.

\subsection{Crown condition assessment - intensive monitoring in 2014 - Level II sample plot Kopaonik}

Crown condition assessment on the Level II sample plot Kopaonik was carried out on April 22, 2014. The assessment included 30 spruce trees, selected for the purpose of annual crown condition monitoring on subplot 2 .

Table 2. (PLT) Data on the plot selected for crown condition assessment, Level

\begin{tabular}{|c|c|c|c|c|c|c|c|c|}
\hline $\begin{array}{c}\text { Sequence } \\
\text { number }\end{array}$ & $\begin{array}{c}\text { Country } \\
\text { code }\end{array}$ & Plot number & $\begin{array}{c}\text { Date of } \\
\text { survey }\end{array}$ & Latitude & Longitude & Altitude & $\begin{array}{c}\text { Team } \\
\text { identification }\end{array}$ & $\begin{array}{c}\text { Other } \\
\text { thervations }\end{array}$ \\
\hline 1 & 67 & 2 & 22.04 .2014 & $+43^{0} 17^{\prime} 30^{\prime \prime}$ & $+20^{\circ} 48^{\prime} 50^{\prime \prime}$ & 35 & & \\
\hline
\end{tabular}

The assessment of crown condition included: defoliation rate, mortalityremoval of trees, tree social status, crown shading, crown visibility, foliage transparency and other observations (Table 3 ).

Tables 3 and 4 show the parameters of crown condition and parameters of damage on the sample plot on Kopaonik in 2014. 
Table 3. (TRC) Crown condition parameters, Level II - Kopaonik

\begin{tabular}{|c|c|c|c|c|c|c|c|c|c|c|c|}
\hline $\begin{array}{c}\text { Sequence } \\
\text { number } \\
\text { of trees }\end{array}$ & $\begin{array}{c}\text { Obse } \\
\text { rvati } \\
\text { on } \\
\text { plot } \\
\text { numb } \\
\text { er }\end{array}$ & $\begin{array}{l}\text { Date of } \\
\text { survey }\end{array}$ & $\begin{array}{c}\text { Tree } \\
\text { number }\end{array}$ & $\begin{array}{l}\text { Tree } \\
\text { species } \\
\text { code }\end{array}$ & $\begin{array}{c}\text { Removal } \\
\text { s \& } \\
\text { mortalit } \\
y\end{array}$ & $\begin{array}{c}\text { Social } \\
\text { class }\end{array}$ & $\begin{array}{l}\text { Crown } \\
\text { shading }\end{array}$ & $\begin{array}{l}\text { Crown } \\
\text { visibility }\end{array}$ & Defoliation & $\begin{array}{c}\text { Foliage } \\
\text { transparency }\end{array}$ & $\begin{array}{c}\text { Other } \\
\text { observations }\end{array}$ \\
\hline 1 & 2 & 220414 & 75 & 118 & 01 & 1 & 2 & 2 & 5 & 15 & U.b. ${ }^{*}$ \\
\hline 2 & 2 & 220414 & 76 & 118 & 01 & 1 & 1 & 2 & 10 & 15 & U.b. ${ }^{*}$ \\
\hline 3 & 2 & 220414 & 78 & 118 & 01 & 1 & 2 & 2 & 15 & 25 & U.b. ${ }^{*}$ \\
\hline 4 & 2 & 220414 & 79 & 118 & 01 & 1 & 1 & 2 & 15 & 20 & U.b. ${ }^{*}$ \\
\hline 5 & 2 & 220414 & 80 & 118 & 01 & 1 & 1 & 2 & 10 & 15 & U.b. ${ }^{*}$ \\
\hline 6 & 2 & 220414 & 85 & 118 & 01 & 1 & 2 & 2 & 15 & 25 & U.b. ${ }^{*}$ \\
\hline 7 & 2 & 220414 & 86 & 118 & 01 & 1 & 3 & 3 & 10 & 20 & U.b. ${ }^{*}$ \\
\hline 8 & 2 & 220414 & 87 & 118 & 01 & 3 & 3 & 3 & 35 & 65 & U.b. * \\
\hline 9 & 2 & 220414 & 88 & 118 & 38 & 5 & 6 & 2 & 100 & 99 & U.b. ${ }^{*}$ \\
\hline 10 & 2 & 220414 & 91 & 118 & 41 & & & & & & \\
\hline 11 & 2 & 220414 & 92 & 118 & 01 & 2 & 3 & 3 & 20 & 30 & U.b. * \\
\hline 12 & 2 & 220414 & 93 & 118 & 01 & 1 & 3 & 3 & 15 & 20 & U.b. ${ }^{*}$ \\
\hline 13 & 2 & 220414 & 94 & 118 & 01 & 3 & 3 & 3 & 40 & 60 & U.b. ${ }^{*}$ \\
\hline 14 & 2 & 220414 & 95 & 118 & 01 & 2 & 3 & 3 & 20 & 30 & U.b. ${ }^{*}$ \\
\hline 15 & 2 & 220414 & 96 & 118 & 01 & 1 & 4 & 4 & 20 & 25 & U.b. ${ }^{*}$ \\
\hline 16 & 2 & 220414 & 97 & 118 & 01 & 1 & 3 & 3 & 15 & 20 & U.b. ${ }^{*}$ \\
\hline 17 & 2 & 220414 & 98 & 118 & 01 & 1 & 3 & 3 & 15 & 20 & U.b. ${ }^{*}$ \\
\hline 18 & 2 & 220414 & 113 & 118 & 01 & 1 & 2 & 2 & 15 & 25 & U.b. ${ }^{*}$ \\
\hline 19 & 2 & 220414 & 114 & 118 & 01 & 1 & 4 & 3 & 20 & 25 & U.b. ${ }^{*}$ \\
\hline 20 & 2 & 220414 & 115 & 118 & 01 & 1 & 3 & 3 & 25 & 30 & U.b. ${ }^{*}$ \\
\hline 21 & 2 & 220414 & 117 & 118 & 01 & 1 & 4 & 3 & 15 & 30 & U.b. ${ }^{*}$ \\
\hline 22 & 2 & 220414 & 118 & 118 & 01 & 1 & 3 & 2 & 20 & 35 & U.b. ${ }^{*}$ \\
\hline 23 & 2 & 220414 & 119 & 118 & 38 & 5 & 6 & 3 & 100 & 99 & U.b. ${ }^{*}$ \\
\hline 24 & 2 & 220414 & 120 & 118 & 01 & 1 & 1 & 2 & 30 & 50 & U.b. ${ }^{*}$ \\
\hline 25 & 2 & 220414 & 121 & 118 & 01 & 1 & 3 & 3 & 20 & 25 & U.b. ${ }^{*}$ \\
\hline 26 & 2 & 220414 & 124 & 118 & 01 & 1 & 2 & 2 & 20 & 30 & U.b. ${ }^{*}$ \\
\hline 27 & 2 & 220414 & 125 & 118 & 38 & 5 & 6 & 3 & 100 & 99 & U.b. ${ }^{*}$ \\
\hline 28 & 2 & 220414 & 126 & 118 & 01 & 1 & 2 & 2 & 10 & 20 & U.b. ${ }^{*}$ \\
\hline 29 & 2 & 220414 & 77 & 118 & 01 & 1 & 3 & 2 & 10 & 25 & U.b. ${ }^{*}$ \\
\hline 30 & 2 & 220414 & 123 & 118 & 01 & 1 & 1 & 1 & 15 & 20 & U.b. * \\
\hline
\end{tabular}

*Usnea barbata

Compared to the previous four years, the percentage of trees with no defoliation increased on the sample plot Kopaonik in 2014. The percentage of dead trees slightly increased, while the percentage of trees with moderate defoliation decreased compared to the previous years. Severe defoliation was not detected in the trees selected for crown condition monitoring in 2014.

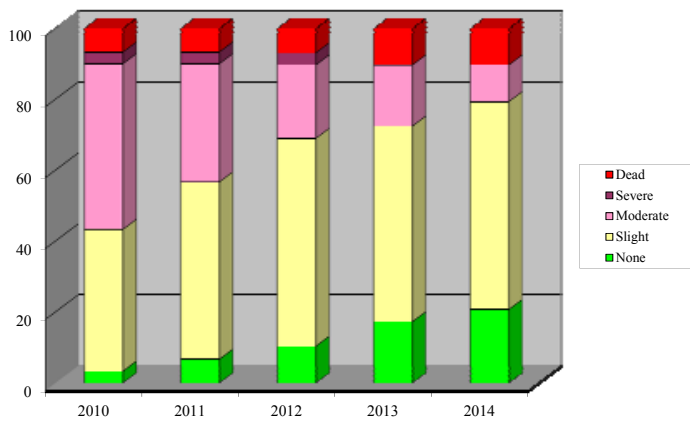

Graph 1. Comparative graphic representation of defoliation in the period from 2010 to 2014 - Level II, Kopaonik 
Table 4. (TRD) Damage parameters, Level II- Kopaonik

\begin{tabular}{|c|c|c|c|c|c|c|c|c|c|c|c|c|}
\hline $\begin{array}{c}\text { Sequence } \\
\text { number } \\
\text { of tree }\end{array}$ & $\begin{array}{l}\text { Observation } \\
\text { plot number }\end{array}$ & $\begin{array}{l}\text { Date of } \\
\text { survey }\end{array}$ & $\begin{array}{c}\text { Tree } \\
\text { number }\end{array}$ & $\begin{array}{c}\text { Specification } \\
\text { of affected } \\
\text { part }\end{array}$ & Symptom & $\begin{array}{l}\text { Specification } \\
\text { of symptom }\end{array}$ & Location & $\begin{array}{c}\text { Age of } \\
\text { damage }\end{array}$ & Cause & $\begin{array}{c}\text { Scientific } \\
\text { name of cause }\end{array}$ & Extent &  \\
\hline 1 & 2 & 220414 & 75 & & & & & & & & & U.b. * \\
\hline 2 & 2 & 220414 & 76 & & & & & & & & & U.b. ${ }^{*}$ \\
\hline 3 & 2 & 220414 & 78 & & & & & & & & & U.b. ${ }^{*}$ \\
\hline 4 & 2 & 220414 & 79 & & & & & & & & & U.b. ${ }^{*}$ \\
\hline 5 & 2 & 220414 & 80 & & & & & & & & & U.b. ${ }^{*}$ \\
\hline 6 & 2 & 220414 & 85 & & & & & & & & & U.b. ${ }^{*}$ \\
\hline 7 & 2 & 220414 & 86 & & & & & & & & & U.b. ${ }^{*}$ \\
\hline 8 & 2 & 220414 & 87 & & & & & & & & & U.b. ${ }^{*}$ \\
\hline 9 & 2 & 220414 & 88 & 32 & 10 & 65 & & 1 & 200 & \begin{tabular}{|c|} 
Pityogenes \\
chalcographus
\end{tabular} & 2 & U.b. ${ }^{*}$ \\
\hline 10 & 2 & 220414 & 91 & & & & & & & & & \\
\hline 11 & 2 & 220414 & 92 & & & & & & & & & U.b. * \\
\hline 12 & 2 & 220414 & 93 & 33 & 17 & 60 & & 3 & 999 & & 3 & U.b. ${ }^{*}$ \\
\hline 13 & 2 & 220414 & 94 & & & & & & & & & U.b. ${ }^{*}$ \\
\hline 14 & 2 & 220414 & 95 & & & & & & & & & U.b. ${ }^{*}$ \\
\hline 15 & 2 & 220414 & 96 & & & & & & & & & U.b. ${ }^{*}$ \\
\hline 16 & 2 & 220414 & 97 & & & & & & & & & U.b. ${ }^{*}$ \\
\hline 17 & 2 & 220414 & 98 & & & & & & & & & U.b. * \\
\hline 18 & 2 & 220414 & 113 & & & & & & & & & U.b. * \\
\hline 19 & 2 & 220414 & 114 & 32 & 10 & 65 & & 1 & 200 & \begin{tabular}{|c|} 
Pityogenes \\
chalcographus
\end{tabular} & 2 & U.b. * \\
\hline 20 & 2 & 220414 & 115 & & & & & & & & & U.b. * \\
\hline 21 & 2 & 220414 & 117 & & & & & & & & & U.b. ${ }^{*}$ \\
\hline 22 & 2 & 220414 & 118 & & & & & & & & & U.b. ${ }^{*}$ \\
\hline 23 & 2 & 220414 & 119 & 32 & 10 & 65 & & 1 & 200 & \begin{tabular}{|c|} 
Pityogenes \\
chalcographus
\end{tabular} & 2 & U.b. ${ }^{*}$ \\
\hline 24 & 2 & 220414 & 120 & & & & & & & & & U.b. ${ }^{*}$ \\
\hline 25 & 2 & 220414 & 121 & & & & & & & & & U.b. ${ }^{*}$ \\
\hline 26 & 2 & 220414 & 124 & & & & & & & & & U.b. ${ }^{*}$ \\
\hline 27 & 2 & 220414 & 125 & 32 & 10 & 65 & & 1 & 200 & \begin{tabular}{|c|} 
Pityogenes \\
chalcographus
\end{tabular} & 2 & U.b. * \\
\hline 28 & 2 & 220414 & 126 & & & & & & & & & U.b. ${ }^{*}$ \\
\hline 29 & 2 & 220414 & 77 & & & & & & & & & U.b. ${ }^{*}$ \\
\hline 30 & 2 & 220414 & 123 & & & & & & & & & U.b. ${ }^{*}$ \\
\hline
\end{tabular}

*Usnea barbata

Health inspection of the Level II sample plot Kopaonik was carried out on April 22, 2014. On that occasion, defoliation was assessed, damage recorded by types and agents and all other relevant observations recorded. The inspection was performed on 30 trees selected within the plot.

It was then observed that 3 trees were dead and infested by bark beetles (trees 88, 119 and 125). Tree 114 was also attacked by bark beetles. Plant diseases were present only in the form of branch decay in tree 113.

\subsection{Crown condition assessment - intensive monitoring in 2014 - Level II sample plot Crni Vrh}

Crown condition assessment on the Level II sample plot Crni Vrh was carried out on June 17,2014. The assessment included 30 beech trees, selected for the purpose of annual crown condition monitoring on subplot 2 . 
Table 5. (PLT) Data on the plot selected for crown condition assessment, Level II, Crni Vrh

\begin{tabular}{|c|c|c|c|c|c|c|c|c|}
\hline $\begin{array}{c}\text { Sequence } \\
\text { number }\end{array}$ & $\begin{array}{c}\text { Country } \\
\text { code }\end{array}$ & Plot number & $\begin{array}{c}\text { Date of } \\
\text { survey }\end{array}$ & Latitude & Longitude & Altitude/Code & $\begin{array}{c}\text { Team } \\
\text { identification }\end{array}$ & $\begin{array}{c}\text { Other } \\
\text { observations }\end{array}$ \\
\hline 1 & 67 & 4 & 17.06 .2014 & $+44^{0} 07^{\prime} 55^{\prime \prime}$ & $+21^{0} 58^{\prime} 38^{\prime \prime}$ & 19 & & \\
\hline
\end{tabular}

Table 6. (TRC) Crown condition parameters, Level II, Crni Vrh

\begin{tabular}{|c|c|c|c|c|c|c|c|c|c|c|c|}
\hline $\mid \begin{array}{c}\text { Sequence } \\
\text { number } \\
\text { of trees }\end{array}$ & $\begin{array}{c}\text { Obse } \\
\text { rvati } \\
\text { on } \\
\text { plot } \\
\text { numb } \\
\text { er }\end{array}$ & $\begin{array}{l}\text { Date of } \\
\text { survey }\end{array}$ & $\begin{array}{c}\text { Tree } \\
\text { number }\end{array}$ & $\begin{array}{c}\text { Tree } \\
\text { specie } \\
\text { s code }\end{array}$ & $\begin{array}{c}\text { Removal } \\
\text { s \& } \\
\text { mortalit } \\
y\end{array}$ & $\begin{array}{c}\text { Social } \\
\text { class }\end{array}$ & $\begin{array}{l}\text { Crown } \\
\text { shading }\end{array}$ & $\begin{array}{l}\text { Crown } \\
\text { visibility }\end{array}$ & Defoliation & $\begin{array}{c}\text { Foliage } \\
\text { transparency }\end{array}$ & $\begin{array}{c}\text { Other } \\
\text { observations }\end{array}$ \\
\hline 1 & 4 & 170614 & 57 & 018 & 01 & 1 & 4 & 2 & 0 & 10 & \\
\hline 2 & 4 & 170614 & 58 & 018 & 01 & 1 & 4 & 2 & 0 & 5 & \\
\hline 3 & 4 & 170614 & 62 & 018 & 01 & 1 & 1 & 1 & 10 & 15 & \\
\hline 4 & 4 & 170614 & 64 & 018 & 01 & 1 & 4 & 1 & 5 & 5 & \\
\hline 5 & 4 & 170614 & 65 & 018 & 01 & 1 & 4 & 1 & 5 & 5 & \\
\hline 6 & 4 & 170614 & 66 & 018 & 01 & 1 & 4 & 1 & 10 & 10 & \\
\hline 7 & 4 & 170614 & 67 & 018 & 01 & 1 & 4 & 2 & 15 & 15 & \\
\hline 8 & 4 & 170614 & 68 & 018 & 01 & 1 & 4 & 2 & 100 & 99 & \\
\hline 9 & 4 & 170614 & 69 & 018 & 01 & 1 & 4 & 2 & 0 & 5 & \\
\hline 10 & 4 & 170614 & 71 & 018 & 01 & 1 & 4 & 2 & 10 & 10 & \\
\hline 11 & 4 & 170614 & 72 & 018 & 01 & 1 & 4 & 2 & 35 & 25 & \\
\hline 12 & 4 & 170614 & 73 & 018 & 01 & 1 & 4 & 1 & 15 & 5 & \\
\hline 13 & 4 & 170614 & 74 & 018 & 01 & 1 & 4 & 1 & 0 & 5 & \\
\hline 14 & 4 & 170614 & 75 & 018 & 01 & 1 & 4 & 1 & 5 & 5 & \\
\hline 15 & 4 & 170614 & 76 & 018 & 01 & 1 & 3 & 1 & 0 & 5 & \\
\hline 16 & 4 & 170614 & 77 & 018 & 01 & 1 & 5 & 1 & 10 & 15 & \\
\hline 17 & 4 & 170614 & 78 & 018 & 01 & 1 & 3 & 1 & 15 & 10 & \\
\hline 18 & 4 & 170614 & 79 & 018 & 01 & 1 & 5 & 1 & 0 & 5 & \\
\hline 19 & 4 & 170614 & 87 & 018 & 01 & 2 & 1 & 1 & 0 & 5 & \\
\hline 20 & 4 & 170614 & 88 & 018 & 01 & 1 & 4 & 1 & 10 & 5 & \\
\hline 21 & 4 & 170614 & 89 & 018 & 01 & 1 & 3 & 1 & 15 & 15 & \\
\hline 22 & 4 & 170614 & 90 & 018 & 01 & 2 & 3 & 1 & 0 & 10 & \\
\hline 23 & 4 & 170614 & 91 & 018 & 01 & 2 & 4 & 1 & 5 & 10 & \\
\hline 24 & 4 & 170614 & 92 & 018 & 01 & 1 & 3 & 1 & 20 & 15 & \\
\hline 25 & 4 & 170614 & 94 & 018 & 01 & 1 & 4 & 2 & 5 & 5 & \\
\hline 26 & 4 & 170614 & 95 & 018 & 01 & 1 & 4 & 1 & 10 & 10 & \\
\hline 27 & 4 & 170614 & 96 & 018 & 01 & 1 & 4 & 1 & 10 & 10 & \\
\hline 28 & 4 & 170614 & 97 & 018 & 01 & 2 & 4 & 2 & 5 & 5 & \\
\hline 29 & 4 & 170614 & 98 & 018 & 01 & 1 & 4 & 1 & 0 & 10 & \\
\hline 30 & 4 & 170614 & 100 & 018 & 01 & 1 & 4 & 2 & 10 & 5 & \\
\hline
\end{tabular}

Defoliation was not recorded in $76.67 \%$ of sample trees (subplot 2), while $16.67 \%$ of trees showed signs of slight defoliation and $3.33 \%$ of moderate defoliation. Strong defoliation was not detected in the trees selected for crown condition monitoring.

The percentage of dead trees and trees with no or slight defoliation on the sample plot Crni Vrh in 2014 was the same as in the previous year. 


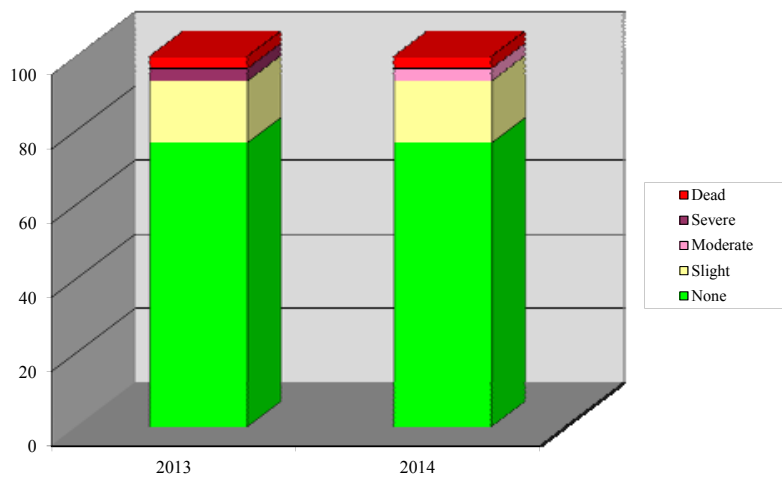

Graph 2. Comparative graphic representation of defoliation in the period 20132014 - Level II, Crni Vrh

Table 7. (TRD) Damage parameters, Level II, Crni Vrh

\begin{tabular}{|c|c|c|c|c|c|c|c|c|c|c|c|c|}
\hline $\begin{array}{l}\text { Sequence } \\
\text { number of } \\
\text { tree }\end{array}$ & $\begin{array}{l}\text { Observatio } \\
\text { n plot } \\
\text { number }\end{array}$ & $\begin{array}{l}\text { Date of } \\
\text { survey }\end{array}$ & $\begin{array}{l}\text { Tree } \\
\text { num } \\
\text { ber }\end{array}$ & $\begin{array}{l}\text { Specification } \\
\text { of affected } \\
\text { part }\end{array}$ & $\begin{array}{l}\text { Sympto } \\
\mathrm{m}\end{array}$ & $\begin{array}{l}\text { Specification } \\
\text { of symptom }\end{array}$ & $\begin{array}{l}\text { Location } \\
\text { in crown }\end{array}$ & $\begin{array}{l}\text { Age of } \\
\text { damage }\end{array}$ & Cause & $\begin{array}{l}\text { Scientific } \\
\text { name of } \\
\text { cause }\end{array}$ & $\begin{array}{l}\text { Exten } \\
\mathrm{t}\end{array}$ & $\begin{array}{l}\text { Other } \\
\text { observatio } \\
\text { ns }\end{array}$ \\
\hline 1 & 4 & 170614 & 57 & & & & & & & & & \\
\hline 2 & 4 & 170614 & 58 & & & & & & & & & \\
\hline 3 & 4 & 170614 & 62 & 14 & 01 & 33 & 3 & 3 & 210 & & 2 & \\
\hline 4 & 4 & 170614 & 64 & & & & & & & & & \\
\hline 5 & 4 & 170614 & 65 & 14 & 01 & 33 & 3 & 3 & 210 & & 2 & \\
\hline 6 & 4 & 170614 & 66 & & & & & & & & & \\
\hline 7 & 4 & 170614 & 67 & 14 & 01 & 33 & 3 & 3 & 210 & & 2 & \\
\hline 8 & 4 & 170614 & 68 & & & & & & & & & \\
\hline 9 & 4 & 170614 & 69 & & & & & & & & & \\
\hline 10 & 4 & 170614 & 71 & & & & & & & & & \\
\hline 11 & 4 & 170614 & 72 & 14 & 01 & 33 & 3 & 3 & 210 & & 2 & \\
\hline 12 & 4 & 170614 & 73 & 14 & 01 & 33 & 3 & 3 & 210 & & 2 & \\
\hline 13 & 4 & 170614 & 74 & & & & & & & & & \\
\hline 14 & 4 & 170614 & 75 & & & & & & & & & \\
\hline 15 & 4 & 170614 & 76 & 32 & 11 & 57 & & 3 & 300 & $\begin{array}{c}\text { Nectria } \\
\text { coccinea }\end{array}$ & 2 & \\
\hline 16 & 4 & 170614 & 77 & & & & & & & & & \\
\hline 17 & 4 & 170614 & 78 & & & & & & & & & \\
\hline 18 & 4 & 170614 & 79 & & & & & & & & & \\
\hline 19 & 4 & 170614 & 87 & 32 & 11 & 57 & & 3 & 300 & $\begin{array}{l}\text { Nectria } \\
\text { coccinea }\end{array}$ & 2 & \\
\hline 20 & 4 & 170614 & 88 & & & & & & & & & \\
\hline 21 & 4 & 170614 & 89 & & & & & & & & & \\
\hline 22 & 4 & 170614 & 90 & & & & & & & & & \\
\hline 23 & 4 & 170614 & 91 & & & & & & & & & \\
\hline 24 & 4 & 170614 & 92 & & & & & & & & & \\
\hline 25 & 4 & 170614 & 94 & & & & & & & & & \\
\hline 26 & 4 & 170614 & 95 & & & & & & & & & \\
\hline 27 & 4 & 170614 & 96 & 14 & 01 & 33 & 3 & 3 & 210 & & 2 & \\
\hline 28 & 4 & 170614 & 97 & & & & & & & & & \\
\hline 29 & 4 & 170614 & 98 & & & & & & & & & \\
\hline 30 & 4 & 170614 & $\begin{array}{c}10 \\
0 \\
\end{array}$ & 14 & 01 & 33 & 3 & 3 & 210 & & 2 & \\
\hline
\end{tabular}

Health inspection of the level II sample plot Crni Vrh was carried out on June 17, 2014.

Plant pathogens affected two trees (76 and 87), on which the presence of the fungus Nectria coccinea uredopustules was registered. Together with the insect Cryptococcus fagisuga it causes a very serious 'beech bark disease'. In addition, 
we recorded wood-rotting fungi on the branches and knots (of trees 62, 65, 67, 72, 73,96 and 100) as well as the central trunk rot (on tree 90).

The damage caused by insects included mainly slight or moderate defoliation, caused probably by gypsy moth, since this year saw the outbreak of gypsy moth in the area.

\subsection{Crown condition assessment - intensive monitoring in 2014 - Level II sample plot Mokra Gora}

Crown condition assessment on the Level II sample plot Mokra Gora was carried out on July 30, 2014. The assessment included 30 Scots pine trees selected for the purpose of annual crown condition monitoring on subplot 2 .

Table 8. (PLT) Data on the plot selected for crown condition assessment, Level II, Mokra Gora

\begin{tabular}{|c|c|c|c|c|c|c|c|c|}
\hline $\begin{array}{c}\text { Sequence } \\
\text { number }\end{array}$ & Country code & Plot number & $\begin{array}{c}\text { Date of } \\
\text { survey }\end{array}$ & Latitude & Longitude & Altitude/Code & $\begin{array}{c}\text { Team } \\
\text { identification }\end{array}$ & $\begin{array}{c}\text { Other } \\
\text { observations }\end{array}$ \\
\hline 1 & 67 & 5 & 30.07 .2014 & $+43^{0} 45^{\prime} 27^{\prime \prime}$ & $+19^{0} 29^{\prime} 00^{\prime \prime}$ & 12 & & \\
\hline
\end{tabular}

Table 9. (TRC) Crown condition parameters, Level II, Mokra Gora

\begin{tabular}{|c|c|c|c|c|c|c|c|c|c|c|c|}
\hline $\begin{array}{c}\text { Sequence } \\
\text { number } \\
\text { of trees }\end{array}$ & $\begin{array}{c}\text { Obse } \\
\text { rvati } \\
\text { on } \\
\text { plot } \\
\text { numb } \\
\text { er }\end{array}$ & $\begin{array}{l}\text { Date of } \\
\text { survey }\end{array}$ & $\begin{array}{c}\text { Tree } \\
\text { number }\end{array}$ & $\begin{array}{c}\text { Tree } \\
\text { specie } \\
\text { s code }\end{array}$ & $\begin{array}{c}\text { Removal } \\
\text { s \& } \\
\text { mortalit } \\
y\end{array}$ & $\begin{array}{c}\text { Social } \\
\text { class }\end{array}$ & $\begin{array}{l}\text { Crown } \\
\text { shading }\end{array}$ & $\begin{array}{c}\text { Crown } \\
\text { visibility }\end{array}$ & Defoliation & $\begin{array}{c}\text { Foliage } \\
\text { transparency }\end{array}$ & $\begin{array}{c}\text { Other } \\
\text { observations }\end{array}$ \\
\hline 1 & 5 & 300714 & 82 & 134 & 1 & 2 & 2 & 1 & 0 & 30 & \\
\hline 2 & 5 & 300714 & 83 & 134 & 1 & 1 & 1 & 1 & 10 & 30 & \\
\hline 3 & 5 & 300714 & 84 & 134 & 1 & 2 & 2 & 1 & 5 & 30 & \\
\hline 4 & 5 & 300714 & 105 & 134 & 1 & 1 & 3 & 1 & 10 & 20 & \\
\hline 5 & 5 & 300714 & 106 & 134 & 1 & 1 & 2 & 1 & 15 & 30 & \\
\hline 6 & 5 & 300714 & 107 & 134 & 1 & 2 & 1 & 1 & 0 & 20 & \\
\hline 7 & 5 & 300714 & 113 & 134 & 1 & 1 & 1 & 1 & 0 & 25 & \\
\hline 8 & 5 & 300714 & 114 & 134 & 1 & 1 & 4 & 1 & 10 & 50 & \\
\hline 9 & 5 & 300714 & 140 & 134 & 1 & 2 & 2 & 1 & 5 & 25 & \\
\hline 10 & 5 & 300714 & 141 & 134 & 1 & 1 & 2 & 1 & 0 & 10 & \\
\hline 11 & 5 & 300714 & 142 & 134 & 1 & 2 & 2 & 1 & 0 & 20 & \\
\hline 12 & 5 & 300714 & 143 & 134 & 1 & 2 & 2 & 1 & 5 & 15 & \\
\hline 13 & 5 & 300714 & 144 & 134 & 1 & 2 & 2 & 1 & 5 & 20 & \\
\hline 14 & 5 & 300714 & 165 & 134 & 1 & 2 & 1 & 1 & 0 & 15 & \\
\hline 15 & 5 & 300714 & 166 & 134 & 1 & 2 & 2 & 1 & 0 & 10 & \\
\hline 16 & 5 & 300714 & 167 & 134 & 1 & 2 & 2 & 1 & 5 & 15 & \\
\hline 17 & 5 & 300714 & 168 & 134 & 1 & 2 & 3 & 1 & 5 & 15 & \\
\hline 18 & 5 & 300714 & 183 & 134 & 1 & 1 & 1 & 1 & 10 & 15 & \\
\hline 19 & 5 & 300714 & 184 & 134 & 1 & 2 & 2 & 1 & 0 & 20 & \\
\hline 20 & 5 & 300714 & 185 & 134 & 1 & 3 & 3 & 2 & 0 & 25 & \\
\hline 21 & 5 & 300714 & 193 & 134 & 1 & 1 & 1 & 1 & 0 & 10 & \\
\hline 22 & 5 & 300714 & 194 & 134 & 1 & 1 & 2 & 1 & 0 & 10 & \\
\hline 23 & 5 & 300714 & 213 & 134 & 1 & 2 & 2 & 1 & 0 & 15 & \\
\hline 24 & 5 & 300714 & 214 & 134 & 1 & 3 & 3 & 1 & 0 & 10 & \\
\hline 25 & 5 & 300714 & 215 & 134 & 1 & 3 & 2 & 1 & 0 & 10 & \\
\hline 26 & 5 & 300714 & 223 & 134 & 1 & 1 & 2 & 1 & 5 & 10 & \\
\hline 27 & 5 & 300714 & 224 & 134 & 1 & 3 & 2 & 1 & 0 & 10 & \\
\hline 28 & 5 & 300714 & 320 & 134 & 1 & 2 & 2 & 1 & 10 & 20 & \\
\hline 29 & 5 & 300714 & 359 & 134 & 1 & 1 & 2 & 1 & 10 & 10 & \\
\hline 30 & 5 & 300714 & 407 & 134 & 1 & 1 & 2 & 1 & 0 & 5 & \\
\hline 31 & 5 & 300714 & 82 & 134 & 1 & 2 & 2 & 1 & 0 & 30 & \\
\hline 32 & 5 & 300714 & 83 & 134 & 1 & 1 & 1 & 1 & 10 & 30 & \\
\hline
\end{tabular}


Defoliation in 2014 was not recorded in $96.67 \%$ of the trees selected for crown condition monitoring (subplot 2) on the Level II sample plot Mokra Gora. Slight defoliation was detected in $3.33 \%$ of sample trees. As in the previous year, strong defoliation was not observed on the sample trees.

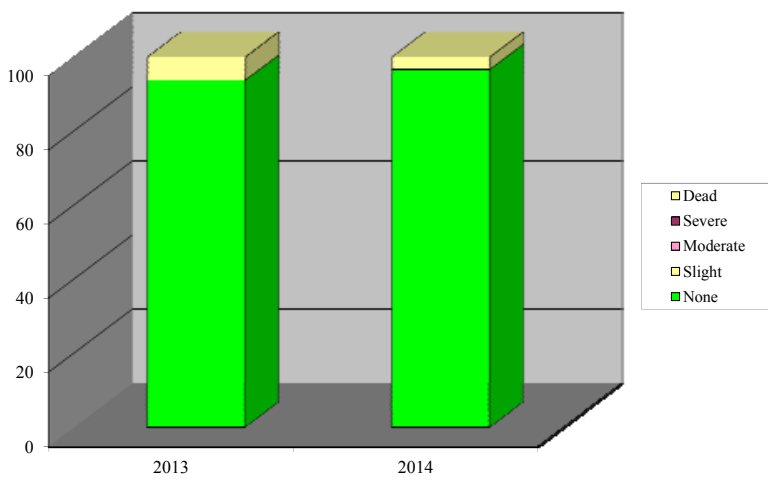

Graph 3. Comparative graphic representation of defoliation in the period from 2013 to 2014 - Level II, Mokra Gora

Health inspection of the Level II sample plot Mokra Gora was carried out on 32 Scots pine trees on the subplot 2.

At the time of inspection, the stand was healthy, free from diseases or pests.

Table 10. (TRD) Damage parameters, Level II, Mokra Gora

\begin{tabular}{|c|c|c|c|c|c|c|c|c|c|c|c|c|}
\hline $\begin{array}{c}\text { Seque } \\
\text { nce } \\
\text { numb } \\
\text { er of } \\
\text { tree }\end{array}$ & $\begin{array}{c}\text { Observ } \\
\text { ation } \\
\text { plot } \\
\text { number }\end{array}$ & Date of survey & $\begin{array}{c}\text { Tree } \\
\text { numbe }\end{array}$ & $\begin{array}{c}\text { Specificat } \\
\text { ion of } \\
\text { affected } \\
\text { part }\end{array}$ & $\begin{array}{c}\text { Sympto } \\
\mathrm{m}\end{array}$ & $\begin{array}{c}\text { Specifica } \\
\text { tion of } \\
\text { symptom }\end{array}$ & $\begin{array}{c}\text { Locatio } \\
\text { in } \\
\text { crown }\end{array}$ & $\begin{array}{c}\text { Age of } \\
\text { damage }\end{array}$ & Cause & $\begin{array}{c}\text { Scientific } \\
\text { name of } \\
\text { cause }\end{array}$ & Extent & $\begin{array}{c}\text { Other } \\
\text { observation } \\
\text { s }\end{array}$ \\
\hline 2 & 5 & 300714 & 82 & & & & & & & & & \\
\hline 3 & 5 & 300714 & 83 & & & & & & & & & \\
\hline 4 & 5 & 300714 & 84 & & & & & & & & & \\
\hline 5 & 5 & 300714 & 105 & & & & & & & & & \\
\hline 6 & 5 & 300714 & 106 & & & & & & & & & \\
\hline 7 & 5 & 300714 & 113 & & & & & & & & & \\
\hline 8 & 5 & 300714 & 114 & & & & & & & & & \\
\hline 9 & 5 & 300714 & 140 & & & & & & & & & \\
\hline 10 & 5 & 300714 & 141 & & & & & & & & & \\
\hline 11 & 5 & 300714 & 142 & & & & & & & & & \\
\hline 12 & 5 & 300714 & 143 & & & & & & & & & \\
\hline 13 & 5 & 300714 & 144 & & & & & & & & & \\
\hline 14 & 5 & 300714 & 165 & & & & & & & & & \\
\hline 15 & 5 & 300714 & 166 & & & & & & & & & \\
\hline 16 & 5 & 300714 & 167 & & & & & & & & & \\
\hline 17 & 5 & 300714 & 168 & & & & & & & & & \\
\hline 18 & 5 & 300714 & 183 & & & & & & & & & \\
\hline 19 & 5 & 300714 & 184 & & & & & & & & & \\
\hline 20 & 5 & 300714 & 185 & & & & & & & & & \\
\hline 21 & 5 & 300714 & 193 & & & & & & & & & \\
\hline 22 & 5 & 300714 & 194 & & & & & & & & & \\
\hline 23 & 5 & 300714 & 213 & & & & & & & & & \\
\hline 24 & 5 & 300714 & 214 & & & & & & & & & \\
\hline 25 & 5 & 300714 & 215 & & & & & & & & & \\
\hline 26 & 5 & 300714 & 223 & & & & & & & & & \\
\hline
\end{tabular}




\begin{tabular}{|c|c|c|c|c|c|c|c|c|c|c|c|c|}
\hline $\begin{array}{c}\text { Seque } \\
\text { nce } \\
\text { numb } \\
\text { er of } \\
\text { tree }\end{array}$ & $\begin{array}{c}\text { Observ } \\
\text { ation } \\
\text { plot } \\
\text { number }\end{array}$ & Date of survey & $\begin{array}{c}\text { Tree } \\
\text { numbe } \\
\mathrm{r}\end{array}$ & $\begin{array}{c}\text { Specificat } \\
\text { ion of } \\
\text { affected } \\
\text { part }\end{array}$ & $\begin{array}{c}\text { Sympto } \\
\mathrm{m}\end{array}$ & $\begin{array}{c}\text { Specifica } \\
\text { tion of } \\
\text { symptom }\end{array}$ & $\begin{array}{c}\text { Locatio } \\
\mathrm{n} \text { in } \\
\text { crown }\end{array}$ & $\begin{array}{c}\text { Age of } \\
\text { damage }\end{array}$ & Cause & $\begin{array}{c}\text { Scientific } \\
\text { name of } \\
\text { cause }\end{array}$ & Extent & $\begin{array}{c}\text { Other } \\
\text { observation } \\
\mathrm{s}\end{array}$ \\
\hline 28 & 5 & 300714 & 224 & & & & & & & & & \\
\hline 29 & 5 & 300714 & 320 & & & & & & & & & \\
\hline 30 & 5 & 300714 & 359 & & & & & & & & & \\
\hline 31 & 5 & 300714 & 407 & & & & & & & & & \\
\hline 32 & 5 & 300714 & 408 & & & & & & & & & \\
\hline
\end{tabular}

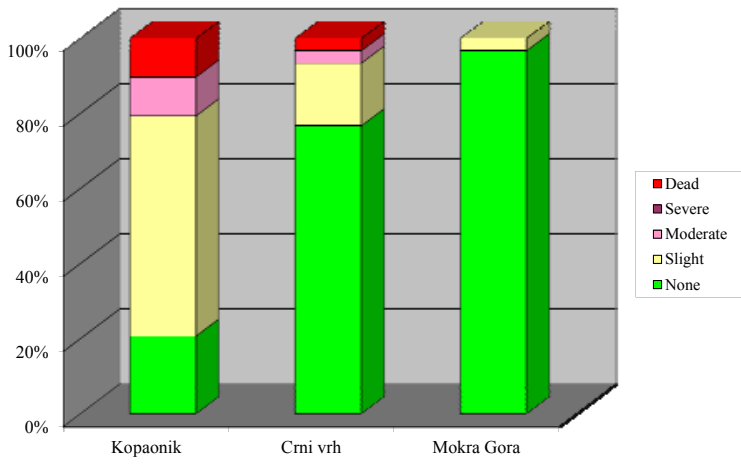

Graph 4. Defoliation rate in 2014 - Level II sample plots

Of all three sites, the highest percentage of trees selected for crown condition monitoring without signs of defoliation was as in 2013 recorded in Mokra Gora. There were no trees with moderate or severe defoliation on this sample plot.

\subsection{GIS application for forest condition monitoring in the Republic of Serbia in 2014}

GIS approach is a general procedure commonly applied in the whole programme of ICP Forests. Large-scale forest areas are monitored at a national level, but the use of this application enables us to represent all data in one common coordinate system. GIS applications are used from the very first stage of monitoring for determining the location of sample plots and marking them in the field using GPS (Global Position System) handheld devices to entering the obtained data into the GIS system, making analyses and models and storing them (Nevenić at al. 2011). 


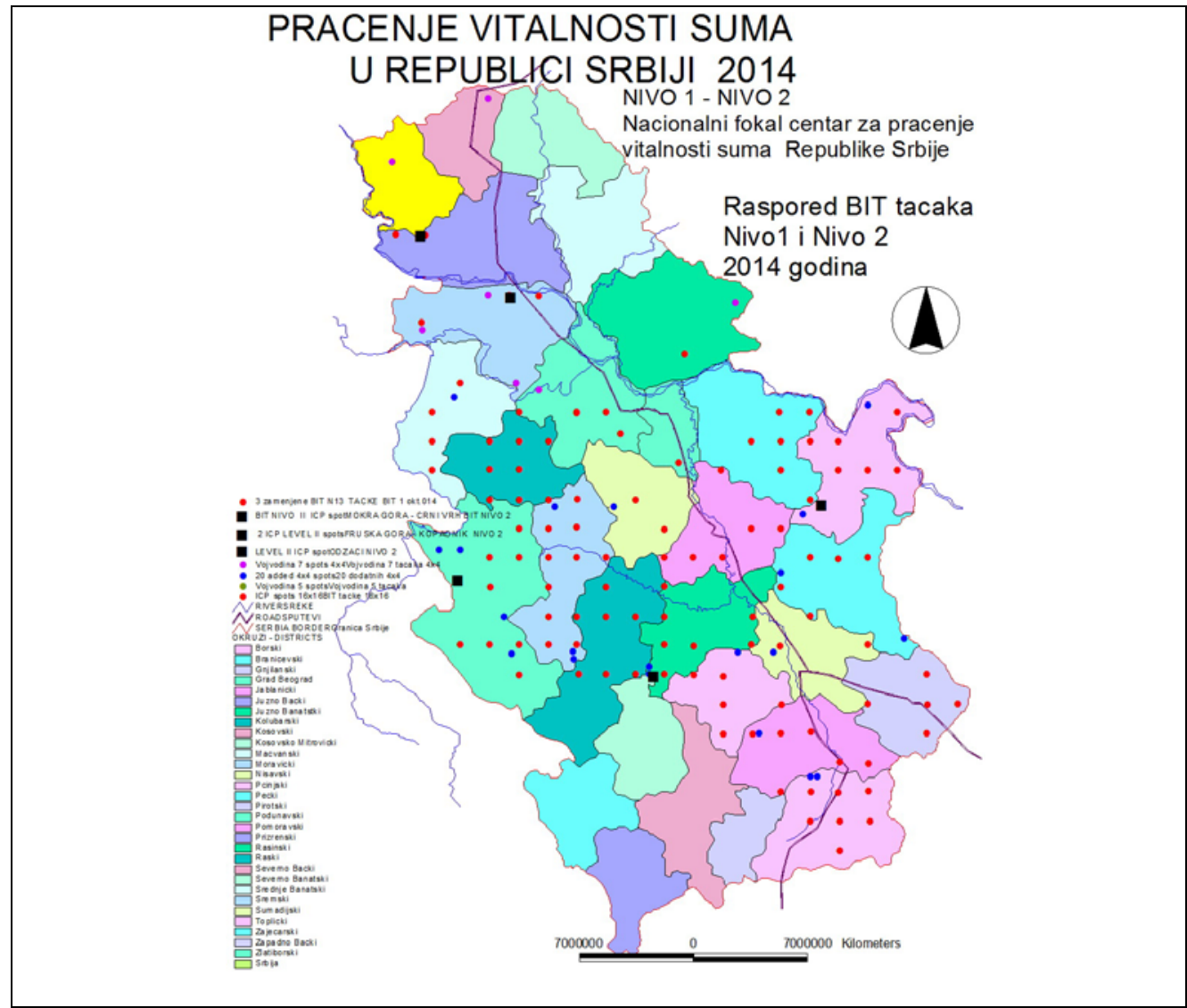

Figure 4. Arrangement of Level and Level II sample plots on the territory of the Republic of Serbia in 2014 - Geographic Information System Application

All Level I and Level II sample plots on the territory of the Republic of Serbia have been entered into the Geographic Information System with the required data and under specific topics. Figure 4 shows the arrangement of Level I and Level II sample plots.

By selecting the appropriate topic or desired data within the appropriate GIS programme, we can obtain a clear representation of all relevant alphanumeric and spatial data.

\section{CONCLUSION}

In 2014, the surveys with parameters that are monitored continuously and annually were carried out on the Level II sample plots Kopaonik, Crni Vrh and Mokra Gora.

In comparison with the previous four years, the percentage of trees with no defoliation increased on the sample plot Kopaonik in 2014. The percentage of dead trees slightly increased, while the percentage of trees with moderate defoliation decreased compared to the previous years. The percentage of trees affected by defoliation of higher intensity (moderate, severe defoliation and dead trees) has 
been decreasing from year to year.

Health inspection of the trees on subplot 2 revealed that out of four trees attacked by bark beetles three were dead and one tree showed signs of branch decay.

Defoliation was not recorded in $76.67 \%$ of the trees on the Level II sample plot on Crni Vrh, while $16.67 \%$ of trees showed signs of slight defoliation and $3.33 \%$ of moderate defoliation. The percentage of trees with no or slight defoliation and the percentage of dead trees was the same as in the previous year.

Plant pathogens affected two trees (76 and 87), on which the presence of the fungus Nectria coccinea uredopustules was registered. In addition, we registered wood-rotting fungi on branches and knots (on 7 trees) as well as the central trunk rot (on 1 tree).

Defoliation in 2014 was not recorded in $96.67 \%$ of the trees on the Level II sample plot Mokra Gora. Slight defoliation affected 3.33\% of sample trees. Strong defoliation was not observed on the selected trees of this sample plot.

At the time of inspection, the stand was healthy, free from diseases or pests.

Generally speaking, the trees on the Level II sample plot Mokra Gora were again least affected by defoliation.

New data are regularly entered into the GIS system of the NFC of Serbia every year and they will make a valuable database for the future scientific research since a multi-year analysis of the vitality of the forest in Serbia is planned to be carried out in a few years.

\section{REFERENCES}

Nevenić, R., Rakonjac, LJ., Orlović, S. (2011): Praćenje uticaj zagađenja vazduha i njegovih efekata u šumskim ekosistemima na teritoriji Republike Srbije - monitoring stanja šuma Nivo I i Nivo II. Monograph. Monitoring and Impact Assessment of Air Pollution and its Effects in Forest Ecosystems on the Territory of the Republic of Serbia. Monograph; NFC Serbia - National Focal Centre. Institute of Forestry, Belgrade. ISBN 978-86-8043928-0. UDK 630*1:502.175(497.11). pp. 1-294.

NEVENIĆ R., BILIBAJKIĆ S., STEFANOVIĆ T., PODUSKA Z., GAGIĆ-SERDAR R., ILIJA ĐORĐEVIĆ., ČEŠLJAR G. (2011): Forest Condition Monitoring of Air Pollution Impact on Forest Ecosystems on the Level II Sample Plot Kopaonik. SUSTAINABLE FORESTRY. Collection volume 63-64 ISSN 1821-1046. UDK 630. pp. 65-76

Svetlana BILIBAJKIĆ, Tomislav STEFANOVIĆ, Radovan NEVENIĆ, Zoran PODUŠKA, Renata GAGIĆ SERDAR, Ilija ĐORĐEVIĆ, Goran ČEŠLJAR, Zoran MILETIĆ (2012): INTENSIVE MONITORING AT LEVEL II TEST PLOT KOPAONIK IN 2012. SUSTAINABLE FORESTRY COLLECTION TOM 65-66. BELGRADE. pp.51-63.

Radovan NEVENIĆ, Svetlana BILIBAJKIĆ, Miroslava MARKOVIĆ, Goran ČEŠLJAR, Tomislav STEFANOVIĆ,Ilija ĐORĐEVIĆ, Zoran PODUŠKA(2013): TREE CROWN CONDITION AT LEVEL II SAMPLE PLOTS KOPAONIK, CRNI VRH AND MOKRA GORA IN 2013. SUSTAINABLE FORESTRY COLLECTION VOLUME 67-68. BELGRADE. pp. 79-94. 
***** (2010): MANUAL on methods and criteria for harmonized sampling, assessment, monitoring and analysis of the effects of air pollution on forests. United Nations Economic Commissions for Europe. Convention on Long-range Transboundary Air Pollution. International Co-operative Programme on Assessment and Monitoring of Air Pollution Effects on Forests (ICP Forests). Programme Coordinating Centre of ICP Forests. Johann Heinrich von Thunen-Institute. Institute for World Forestry, Hamburg, Germany. ISBN 978-3926301-01-1. www.icp-forests.org/Manual.htm

***** (2010a): Manual on methods and criteria for harmonized sampling, assessment, monitoring and analysis of the effects of air pollution on forests - Parts I, II, IX, V, VII, VIII, IX, XVII; ISBN 978-3-926301-01-1, Edited in 2010

***** (2010d): Europe's Forests 1985-2010. 25 Years of Monitoring Forest Condition by ICP Forests. Hohann Heinrich von Thuunen - Institute, Institute for World Forestry. PCC of ICP Forests, Hamburg, Germany.

***** Forms and Explanatary Items to be applied for data submission 2013 onwards Version n8 Last update: 16 May 2013. http://www.icp-forests.org/page/data-submission

Google 1 - http://www.icp-forests.org/Manual.htm

Google 2 - http://www.icp-forests.org/ 